\title{
Near-Complete Photon Spin Selectivity in a Metasurface of Anisotropic Plasmonic Antennas
}

\begin{abstract}
Robin Ogier, Yurui Fang, Mikael Käll, ${ }^{*}$ and Mikael Svedendahl ${ }^{\dagger}$
Department of Applied Physics, Chalmers University of Technology, 41296 Göteborg, Sweden (Received 30 March 2015; revised manuscript received 4 September 2015; published 4 November 2015)

Simple chiroptically active metamaterials are difficult to realize in practice but could pave the way for a range of important applications, such as sensitive optical biosensors, asymmetric catalysis, and novel polarization manipulation devices. We show that a metasurface based on a random arrangement of anisotropic but aligned gold nanoparticles can exhibit an almost perfect selectivity towards incident photon spin for evanescent excitation with visible to near-infrared light. The experimentally attained reflection contrast between left- and right-handed circularly polarized light peaks at $\sim 90 \%$, in excellent agreement with analytical theory. These results are important for the development of future photonic and plasmonic polarization-based technologies.
\end{abstract}

DOI: 10.1103/PhysRevX.5.041019

\section{INTRODUCTION}

A chiroptically active structure preferentially absorbs or scatters light depending on photon spin; that is, it can select between light that is left-handed circular polarized (LHCP) and right-handed circular polarized (RHCP). Chiroptical effects are of significant importance for the analysis of molecular enantiomers, such as DNA, enantioselective biomolecular interactions, and drug development, but the photonspin-dependent light-matter interaction is generally very weak in natural materials. This has motivated the development of a wide range of nanostructures and metamaterials that exhibit enhanced optical chirality [1-5]. Such structures could potentially enable novel and more sensitive molecular sensing technologies, but could also be used in photonics components, such as lasers, filters, or optical communication devices that utilize the handedness of light [1-5].

Pronounced chiroptical responses have been demonstrated for a number of quasi-three-dimensional chiral metamaterials and phase-gradient metasurfaces built from plasmonic nanostructures [5-13], but the fabrication of such structures is challenging because of the high precision and reproducibility needed to properly position the meta-atoms in 3D over macroscopic distances. A simpler approach towards high photon spin selectivity is to use so-called extrinsic chirality based on anisotropic achiral metasurfaces that are tilted with respect to the plane of incidence. Chirality is then a consequence of including the wave

\footnotetext{
*mikael.kall@chalmers.se

†mikael.svedendahl@chalmers.se
}

Published by the American Physical Society under the terms of the Creative Commons Attribution 3.0 License. Further distribution of this work must maintain attribution to the author(s) and the published article's title, journal citation, and DOI.
Subject Areas: Metamaterials, Plasmonics

vector of light in the chiral triad $[14,15]$. The anisotropy can be due either to the geometry of individual meta-atoms or to their positioning within the surface layer [15-18]. However, a facile and effective chiroptical metasurface for the visible to near-infrared wavelength range has not yet been realized.

Evanescent, or total internal reflection, excitation has been shown to lead to dramatic chiroptical effects, such as highly directional photon routing and rotation of small particles [19-24]. We hypothesize that extrinsic chirality in anisotropic plasmonic metasurfaces could be boosted by evanescent excitation. The idea, illustrated in Fig. 1 [25], is that the evanescent illumination geometry results in a helicity-dependent electric-field orientation in the plane of the surface. It should then be possible to orient a surfacebound anisotropic nanoparticle such that it is excited by RHCP light but not by LHCP light, or vice versa. This
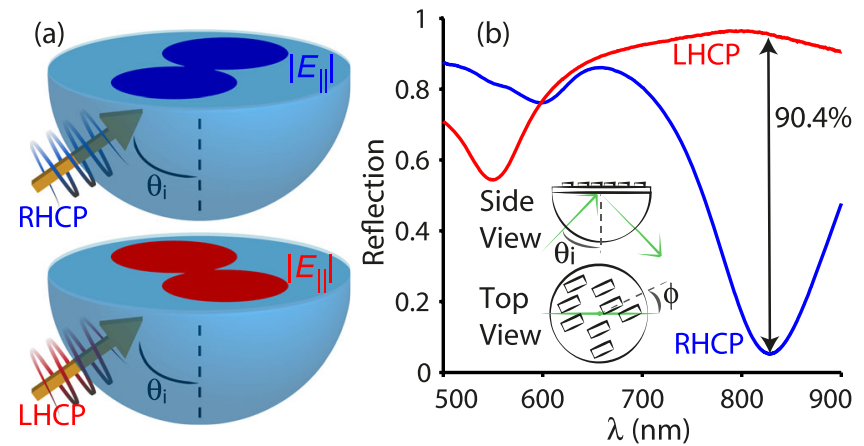

FIG. 1. (a) The in-plane electric field amplitudes $E_{\|}$calculated using Fresnel coefficients of an air-glass boundary at $\theta_{i}=$ $45^{\circ}>\theta_{c} \approx 42^{\circ}$ incidence, using RHCP and LHCP illumination. (b) Reflection measurements from an array of anisotropic gold nanoparticles, under RHCP and LHCP illumination, at $\theta_{i}=45^{\circ}$ and optimal sample orientation $\phi=25^{\circ}$. The insets in (b) define the incidence angle $\theta_{i}$ and the nanoparticle orientation $\phi$. 
should in turn result in a strongly photon-spin-dependent reflectivity. To experimentally verify this hypothesis, we fabricate ultrathin macroscopic layers of elongated gold particles with well-defined anisotropic polarizabilities. The main result is shown in Fig. 1(b). When illuminated slightly above the critical angle, for which no transmission is allowed, and for incidence wavelengths close to the main plasmon resonance of the particles $\left(\lambda_{r} \approx 822 \mathrm{~nm}\right)$, the metasurface can be oriented to absorb $96 \%$ of incident RHCP light while the perpendicular photon spin is almost completely reflected $\left(R_{\mathrm{LHCP}}=94 \%\right.$ versus $\left.R_{\mathrm{RHCP}}=4 \%\right)$. This dramatic photon spin selectivity effect depends on both the angle of incidence $\theta_{i}$ and the orientation of the nanoparticles with respect to the plane of incidence $\phi$. Importantly, analytical theory predicts that the contrast could even be very close to $100 \%$ for optimized structures and illumination configurations. We also find that the optical chirality of the near fields around the nanoparticles are locally enhanced and highly dependent on incident photon spin, although the surface-averaged chiroptical enhancement is modest. Finally, we investigate the polarization state of the reflected light and find that the degree of circular polarization is large under RHCP and LHCP illumination as well as for most elliptical polarizations states. Interestingly, a distinct polarization-phase singularity is found for a narrow range of elliptical polarizations. These results could pave the way for much easier polarization manipulation and chiroptical control, which could in turn lead to new applications in many fields of optics, photonics, and spectroscopy.

\section{RESULTS}

\section{A. Samples}

The samples are fabricated by a variant of hole-mask colloidal lithography [26]. In brief, negatively charged latex beads $(\varnothing=80 \mathrm{~nm})$, which self-assemble on appropriate surfaces with characteristic short-range order due to electrostatic forces, are drop-casted on a glass cover slip covered with a 230-nm-thick layer of polymethyl methacrylate (PMMA). A thin gold mask layer is evaporated on top of the sample surface, after which the beads are removed by tape stripping. After a 5-min oxygen plasma etching process, during which the underlying PMMA layer is overetched, a large glass surface area is made available for deposition through the holes in the gold mask. Elongated gold nanoparticles can then be formed by depositing gold while the evaporation angle is varied continuously $\left( \pm 17^{\circ}\right)$. Mask lift-off and cleaning finalize the sample fabrication procedure. Figures 2(a) and 2(b) show AFM and SEM images of sections of the array. The nanoparticles are $175 \pm 10 \mathrm{~nm}$ long, $75 \pm 5 \mathrm{~nm}$ wide, and $\sim 35 \mathrm{~nm}$ tall. Transmission spectra, taken at normal incidence, reveal distinct single-plasmon resonances when the long or the short in-plane axes of the nanoparticles are lined
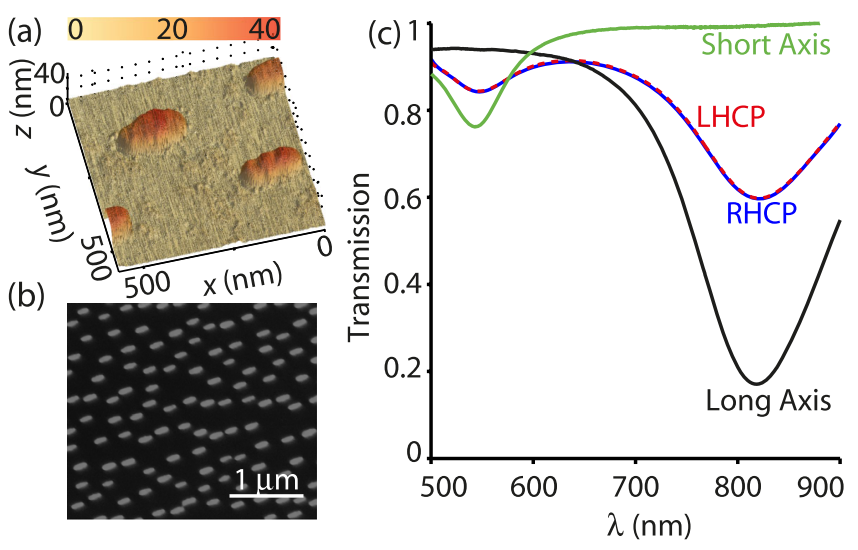

FIG. 2. Sample properties. (a) Atomic force micrograph and (b) scanning electron micrograph of the elongated gold nanoparticles. (c) Optical properties of the array measured at normal incidence $\left(\theta_{i}=0^{\circ}\right)$ displaying the in-plane short- and long-axis resonances and the responses for circularly polarized light.

up with the incident polarization [Fig. 2(c)]. These results demonstrate that the sample possesses a significant and well-defined in-plane anisotropy. However, the sample is not chiral, as confirmed by the identical transmission spectra obtained using LHCP and RHCP, in accordance with what is expected from basic symmetry arguments of the nanoparticles' geometry.

\section{B. Optical characterization}

The chiroptical response of the sample is investigated by placing it in optical contact with a large hemispherical glass prism using index-matching oil and illuminating it from the glass side at an off-normal incidence angle. The polarization state of the incident collimated beam of white light (Ocean Optics, HL-2000-HP) is controlled by a broadband polarizer (Thorlabs, GTH10M) and a quarter-wave Fresnel rhomb (Thorlabs, FR600QM). Reflection spectra are acquired using a fiber-coupled spectrometer (B\&W Tek, BRC711E).

The difference in reflection between RHCP and LHCP light, $\Delta R=R_{\mathrm{RHCP}}-R_{\mathrm{LHCP}}$, exhibits distinct behaviors above and below the critical angle of the interface, $\theta_{c} \approx$ $42^{\circ}$ [Fig. 3(a)]. Below $\theta_{c}, \Delta R$ is relatively small and spectrally broad, with a node close to $\lambda_{r}$. In contrast, when $\theta_{i}>\theta_{c}, \Delta R$ is significantly enhanced with a peak at $\lambda_{r}$.

In the following, we focus on the optical properties for an incidence angle of $\theta_{i}=45^{\circ}$, but similar behavior is found also for other $\theta_{i}>\theta_{c}$ [25]. We use a right-angle prism for these measurements, since this yields equal amplitudes for the $p$ - and $s$-polarized light component hitting the sample surface. The extinction of RHCP is found to be greatly enhanced near $\lambda_{r}$ at $\phi=25^{\circ}$, while the same response is found for LHCP at $\phi=155^{\circ}=-25^{\circ}$ [Figs. 3(b) and 3(c)]. Rotating the sample from $\phi=25^{\circ}$ to $155^{\circ}$ results in a complete mirrored behavior between the responses of LHCP and RHCP. An opposite trend is found near the 


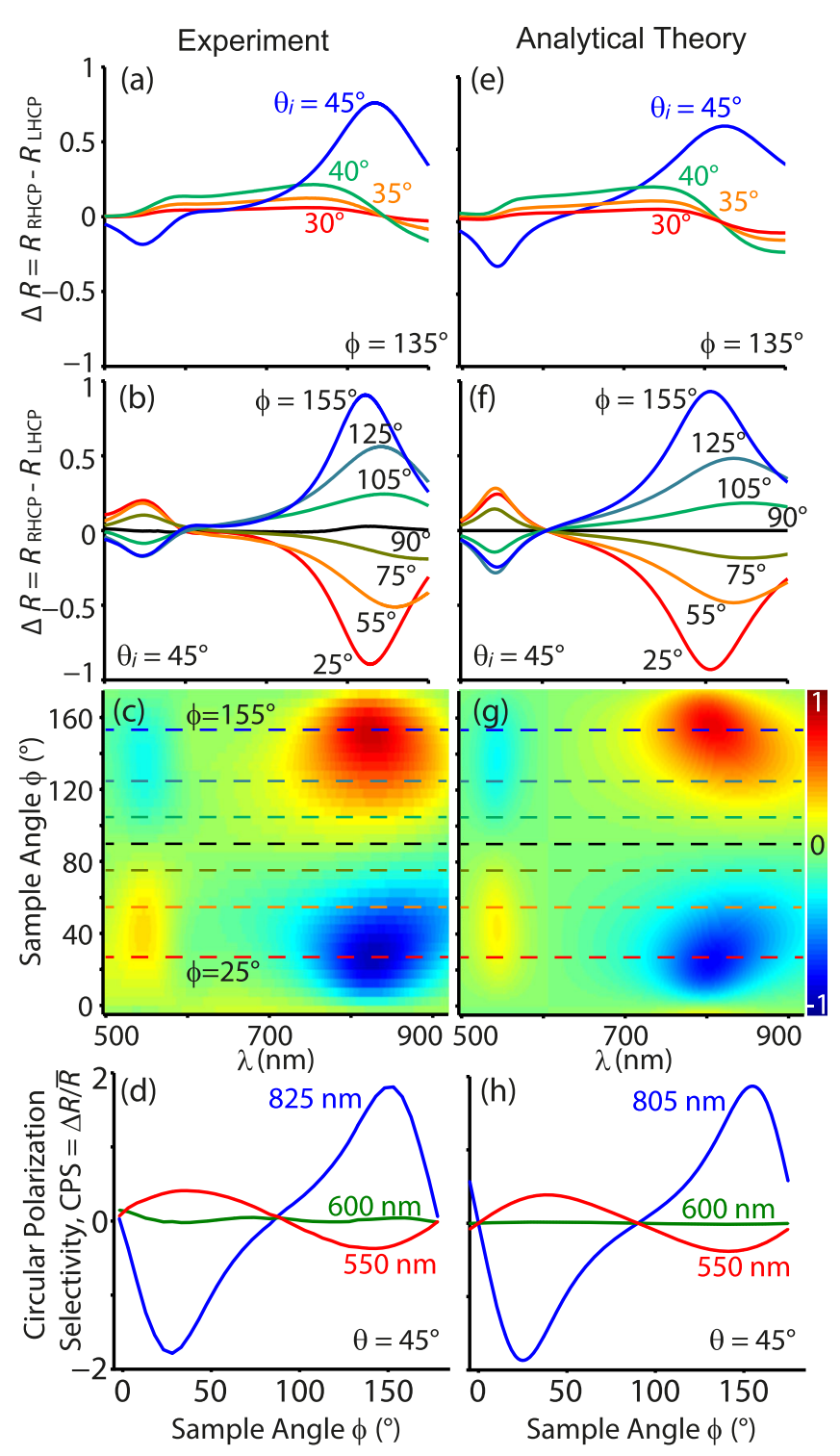

FIG. 3. Helicity-dependent reflections as function of $\theta_{i}$ and $\phi$. (a) $\Delta R$ at $\phi=135^{\circ}$ for varying $\theta_{i}$ below and above $\theta_{c}$. (b),(c) $\Delta R$ at $\theta_{i}=45^{\circ}$ with varying $\phi$. (d) The CPS for the two in-plane resonance wavelengths $(550$ and $825 \mathrm{~nm}$ ) and $600 \mathrm{~nm}$, as found from (c). (e)-(h) Analytical theory based on two in-plane orthogonal dipoles.

short-axis resonance. Note that there is no spectral difference between $\phi=0^{\circ}$ and $90^{\circ}$. The system and its mirror image are identical when one of the symmetry axes of the sample is lined up with the plane of incidence, which thus corresponds to a vanishing extrinsic chirality.

Figure 3(d) shows the circular polarization selectivity, $\mathrm{CPS}=(\Delta R / \bar{R})$, that is, the ratio between the differential and average reflections. The CPS is found to be maximized at $\lambda=\lambda_{r}$ and $\phi= \pm 25^{\circ}$, for which we find CPS $=\mp 1.8$. This almost reaches perfect spin selectivity, which corresponds to $\mathrm{CPS}= \pm 2$. The metasurface can thus preferentially select one circular polarization if illuminated with linearly polarized light with the right wavelength and incidence angle.

\section{Analytical theory}

We have previously modeled reflections from isotropic hole-mask colloidal lithography samples using so-called modified Fresnel equations [27-31]. However, these may be extended to also encompass anisotropic nanostructures. In the following, we neglect the out-of-plane component of the nanoparticle's polarizability for simplicity, since this component plays little or no role in the observed phenomena, but similar equations can also be derived for this case. Briefly, we rewrite the boundary equations of the interface by including a surface polarization, $\vec{P}^{\text {sur }}=\rho \alpha \vec{E}_{\|}$. Here, $\alpha$ and $\rho$ are the nanoparticle in-plane polarizability and surface density, respectively, and $\vec{E}_{\|}$is the in-plane electric field. For isotropic nanoparticles, the reflection coefficients are

$$
\begin{aligned}
& r_{p}=\frac{d_{p}}{N_{p}}=\frac{n_{t} \cos \theta_{i}-n_{i} \cos \theta_{t}+i \frac{\omega}{c} \rho \alpha \cos \theta_{i} \cos \theta_{t}}{n_{t} \cos \theta_{i}+n_{i} \cos \theta_{t}-i \frac{\omega}{c} \rho \alpha \cos \theta_{i} \cos \theta_{t}} \\
& r_{s}=\frac{d_{s}}{N_{s}}=\frac{n_{i} \cos \theta_{i}-n_{t} \cos \theta_{t}+i \frac{\omega}{c} \rho \alpha}{n_{i} \cos \theta_{i}+n_{t} \cos \theta_{t}-i \frac{\omega}{c} \rho \alpha},
\end{aligned}
$$

where $n_{i, t}$ is the refractive index of the incident and transmission media, respectively, $\theta_{t}$ is the angle of refraction, and $\omega / c$ is the wave number. For anisotropic nanoparticles, these equations should be modified and coupled through $\theta_{i}, \phi$, and the anisotropy of the nanostructures. Because of the large spectral separation between the longand short-axis resonances [Fig. 2(c)], and for the readability of the following equations, we focus on the long-axis resonance. A full derivation, including both in-plane resonances, can be found in the Supplemental Material Sec. S2 [25].

An incident $p$-polarized wave has nonzero $x$ and $z$ electric-field components, while the $s$-polarized electric fields are defined along $y . \vec{P}^{\text {sur }}$ can then be written as

$$
\begin{aligned}
& P_{x}^{\text {sur }}=\rho \alpha\left(\cos ^{2} \phi E_{x}+\cos \phi \sin \phi E_{y}\right), \\
& P_{y}^{\text {sur }}=\rho \alpha\left(\cos \phi \sin \phi E_{x}+\sin ^{2} \phi E_{y}\right) .
\end{aligned}
$$

If the long axis of the nanoparticle is aligned with either the $x$ or the $y$ axis, $P_{x}^{\text {sur }}$ and $P_{y}^{\text {sur }}$ are uncoupled. However, for all other orientations, an excitation along $x$ will result in an effective surface polarization along $y$, and vice versa. Even though it might seem beneficial to derive reflection coefficients in the basis of circular polarizations, it is much simpler and more intuitive to solve these equations using $p$ and $s$ polarizations. $\vec{P}^{\text {sur }}$ leads to the following modified Fresnel equations: 


$$
\begin{aligned}
r_{p p} & =\frac{N_{s} d_{p}-\left(\frac{\omega}{c} \rho \alpha \sin \phi \cos \phi\right)^{2} \cos \theta_{i} \cos \theta_{t}}{N_{p} N_{s}-\left(\frac{\omega}{c} \rho \alpha \sin \phi \cos \phi\right)^{2} \cos \theta_{i} \cos \theta_{t}} \\
r_{s s} & =\frac{N_{p} d_{s}-\left(\frac{\omega}{c} \rho \alpha \sin \phi \cos \phi\right)^{2} \cos \theta_{i} \cos \theta_{t}}{N_{p} N_{s}-\left(\frac{\omega}{c} \rho \alpha \sin \phi \cos \phi\right)^{2} \cos \theta_{i} \cos \theta_{t}}, \\
r_{p s} & =-r_{s p}=\frac{2 i n_{i} \cos \theta_{i} \cos \theta_{t} \frac{\omega}{c} \rho \alpha \sin \phi \cos \phi}{N_{p} N_{s}-\left(\frac{\omega}{c} \rho \alpha \sin \phi \cos \phi\right)^{2} \cos \theta_{i} \cos \theta_{t}} .
\end{aligned}
$$

Here, $\rho \alpha$ in $N_{p, s}$ and $d_{p, s}$ should be replaced by $\rho \alpha \cos ^{2} \phi$ and $\rho \alpha \sin ^{2} \phi$, in accordance with Eq. (2). $r_{p p, s s}$ denote reflections of maintained linear polarization, while $r_{p s}$, for example, is the conversion from $p$ to $s$ polarization. The reflections using RHCP and LHCP incident light can be written as $R_{\mathrm{RHCP}}=\frac{1}{2}\left(\left|r_{s s}+i r_{p s}\right|^{2}+\left|r_{s p}+i r_{p p}\right|^{2}\right)$ and $R_{\mathrm{LHCP}}=\frac{1}{2}\left(\left|r_{s s}-i r_{p s}\right|^{2}+\left|r_{s p}-i r_{p p}\right|^{2}\right)$, respectively. If $\alpha$ and $\rho$ are known, then $R_{\mathrm{RHCP}}$ and $R_{\mathrm{LHCP}}$ can effectively be modeled using Eqs. (1)-(3).

In previous studies, we modeled the polarizabilities of isotropic nanoparticles as Lorentzian resonances calculated using a quasistatic approximation of elongated nanoparticles [28-31]. Similarly, the in-plane polarizabilities of the nanoparticles were approximated using the nanoparticle geometry in Fig. 2(a), and the optical properties of the metasurface were subsequently calculated using a particle surface density of $\rho=11.6 \mu \mathrm{m}^{-2}$ and Eqs. (1)-(3) [25]. Figures 3(e)-3(h) show the response of the system using two orthogonally oriented in-plane resonances located at 550 and $825 \mathrm{~nm}$. The similarity between the experimental and analytical data is striking.

\section{Origin of spin selectivity}

Although the large spin selectivity results from the optical far-field properties of the metasurface as a whole, the basic origin of the effect can be understood only by studying the response of a single anisotropic nanoparticle. Figure 4(a) shows finite elements method simulations of the extinction cross section of a single elongated gold nanoparticle, on glass in air, illuminated at $\theta_{i}=45^{\circ}$ and oriented with the long axis along $\phi=25^{\circ}$, that is, corresponding to the optimum conditions shown in Fig. 3. The long-axis plasmon turns out to be excited about 25 times more efficiently with RHCP compared to LHCP illumination. This large excitation contrast is a consequence of the phase difference between the transmitted $p$ - and $s$-polarized components in the plane of the particle for $\theta_{i}>\theta_{c}$. To illustrate this effect, we calculate the field amplitude projected along $\phi$ at $\theta_{i}=45^{\circ}$ with LHCP and RHCP illumination, $\left|E_{\mathrm{LHCP}}(\phi)\right|$ and $\left|E_{\mathrm{RHCP}}(\phi)\right|$, using the Fresnel coefficients of a bare glass-air boundary. $\mid E_{\mathrm{LHCP}}$ or RHCP$(\phi) \mid$ thus represents the field that would excite an in-plane resonance of an anisotropic plasmonic nanoparticle oriented along $\phi$. The field amplitudes are identical if $\theta_{i}<\theta_{c}$ because the phase shift

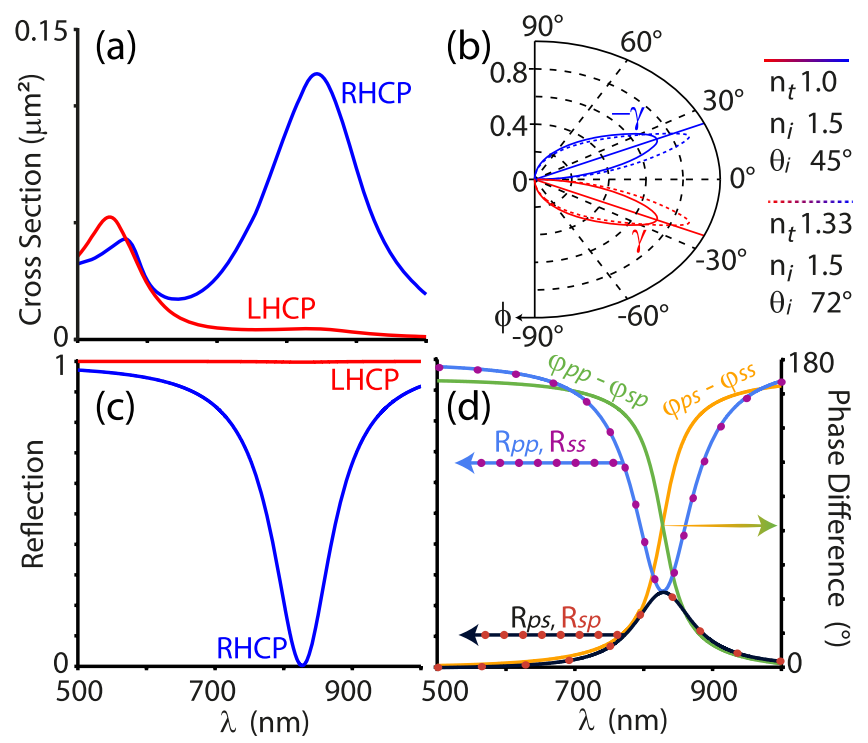

FIG. 4. Origin of the spin selectivity. (a) Finite elements method calculation of the extinction cross section of a single elongated gold nanoparticle, when illuminated at $\theta_{i}=45^{\circ}$ and $\phi=25^{\circ}$. (b) The excitation contrast $\gamma$, see Eq. (4), for a glass-air and glass-water interface. (c) Calculated RHCP and LHCP reflection spectra with optimized $\rho \alpha$ for the glass-water configuration in (b), showing 99\% spin selectivity. (d) Reflection matrix components with the same parameters as (c).

between the $p$ - and $s$-polarized components of the transmitted wave is then either $0^{\circ}$ or $180^{\circ}$. However, this is not the case for $\theta_{i}>\theta_{c}$, and the superposition of the two components along $\phi$ therefore results in varying degrees of constructive or destructive interference depending on the helicity of the incident field [Fig. 4(a)] [25]. Thus, the longaxis resonance of the nanoparticle will be excited with dramatically varying efficiency depending on the handedness of illumination. We can quantify this effect by defining an excitation-field contrast along $\phi$ between RHCP and LHCP illumination:

$$
\gamma(\phi)=\frac{\left|E_{\mathrm{LHCP}}(\phi)\right|-\left|E_{\mathrm{RHCP}}(\phi)\right|}{\left|E_{\mathrm{LHCP}}(\phi)\right|+\mid E_{\mathrm{RHCP}}(\phi)} .
$$

Figure 4(b) shows $\gamma$ as a function of $\phi$ for $\theta_{i}=45^{\circ}$ in the case of the glass-air interface. The highest contrast is found for $\phi= \pm 25^{\circ}$, in agreement with the experimental data in Fig. 3.

We now briefly consider the situation when the air medium around the particles is exchanged to water, since this case has important practical relevance in, for example, optical biosensing applications. Furthermore, by scanning the parameter space, we find that the glass-water configuration can produce near perfect reflection contrast, that is, $R_{\mathrm{RHCP}}=0$ and $R_{\mathrm{LHCP}}=1$, or vice versa, under optimal conditions. In order for this to occur, the two in-plane resonances need to be well separated and the oscillator 
strength of the layer $\rho \alpha$ has to be large enough. For a $\rho \alpha$ only $\sim 16 \%$ larger than the one used to model the experimental data in Figs. 3(e)-3(h), which is easy to achieve in practice, the optimum incidence angle is found to be $\theta_{i}=72^{\circ}$, and the corresponding optimal $\phi$, which thus maximize the excitation contrast Eq. (4), is found to be $\phi \approx 20^{\circ}$ [Fig. 4(b), dashed lines]. These conditions lead to the reflection spectra shown in Fig. 4(c), which shows close to $100 \%$ reflection contrast. We note that to simultaneously obtain $R_{\mathrm{RHCP}}=0$ and $R_{\mathrm{LHCP}}=1$, the modified Fresnel coefficients must satisfy $R_{p p, p s, s, s p}=25 \%$ and there must be a $90^{\circ}$ phase difference between the off-diagonal and diagonal components $\left(\varphi_{p p}-\varphi_{s p}\right.$ and $\left.\varphi_{p s}-\varphi_{s s}\right)$, as shown in Fig. 4(d).

\section{E. Near-field properties}

As expected, the large contrast in excitation efficiency between RHCP and LHCP light at the long-axis resonance (Fig. 4) results in a correspondingly large difference in near-field enhancement around a particle [Figs. 5(a) and 5(b)] [25]. In relation to this effect, it is interesting to also investigate the polarization state of the near fields because it has been suggested that a large so-called optical chirality parameter, $C=-\left(\varepsilon_{0} \omega / 2\right) \operatorname{Im}\left(\vec{E}^{*} \cdot \vec{B}\right)$, might enhance the circular dichroism of chiral molecules residing close to the particle surface [2,32], which could be of great interest for bioapplications of chiral metasurfaces. As shown in Figs. 5(c) and 5(d), illuminating the nanoparticles with the circular polarization state that generates strong local field enhancement also results in enhanced optical chirality in the vicinity of the nanoparticle, while the opposite handedness results in significantly lower values. Note here that the

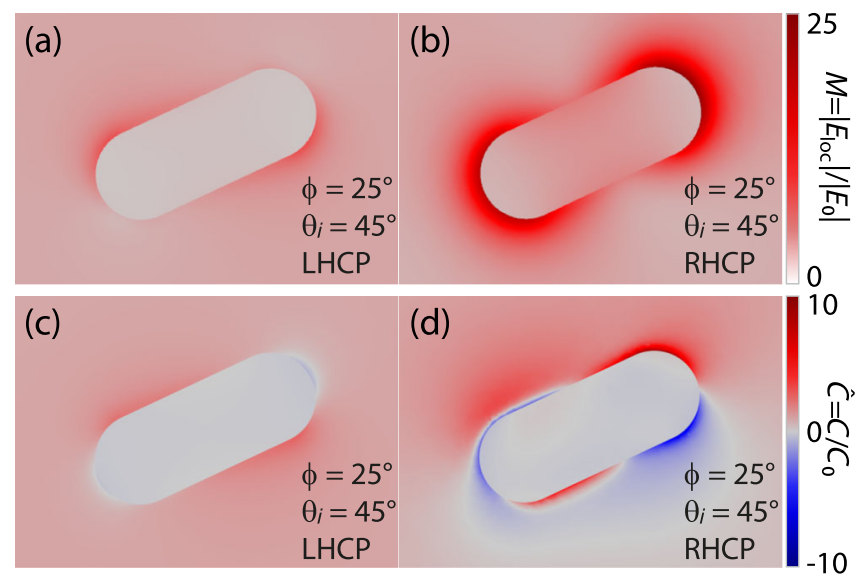

FIG. 5. Near-field properties at the main plasmon resonance wavelength. Calculated near-field enhancement $M=\left|E_{\text {loc }}\right| /\left|E_{0}\right|$ under (a) LHCP and (b) RHCP illumination and the optical chirality enhancement $\hat{C}=C / C_{0}$ under (c) LHCP and (d) RHCP illumination. All figures represent the case of $\theta_{i}=45^{\circ}$ and $\phi=25^{\circ}$, and all data are from a plane $10 \mathrm{~nm}$ above the glassair interface. $C_{0}$ is the optical activity without any nanoparticle. optical chirality $C$ in Figs. 5(c) and 5(d) has been normalized to the case without any nanostructure, $C_{0}$. Note also that the illumination of an elongated nanoparticle at $\theta_{i}>\theta_{c}$ makes $C$ heavily dependent on $\phi$. However, the surface-averaged enhancement of $C$ is low and this might complicate possible future applications based on chiral near fields, as has been discussed for several other achiral nanostructures [2,33-36].

\section{F. Polarization state of the reflected wave}

We now consider the polarization properties of the reflected beam in the experimental system. Figure 6 shows the circular polarization components of the spectra in Fig. 1(b), that is, in the case of $\theta_{i}=45^{\circ}$ and $\phi=25^{\circ}$. Here, the subindex denotes the incident and reflected helicity in the form $R_{\mathrm{in}, \text { out }}$ (the RHCP-reflected component from the surface illuminated with LHCP light is denoted $R_{L R}$, for example). These components can easily be calculated from Eq. (3) using

$$
\begin{aligned}
& R_{R L}=R_{L R}=\frac{1}{4}\left|r_{p p}-r_{s s}\right|^{2}, \\
& R_{R R}=\frac{1}{4}\left|r_{p p}+r_{s s}+2 i r_{s p}\right|^{2}, \\
& R_{L L}=\frac{1}{4}\left|r_{p p}+r_{s s}-2 i r_{s p}\right|^{2} .
\end{aligned}
$$

Focusing on the properties at $\lambda_{r}$, we see that the $R_{R R}$ component approaches zero while the $R_{R L}$ component shows a reflection of $\sim 4 \%$. The reflected polarization state is thus altered from the illumination state, in this case from RHCP to mainly LHCP. On the other hand, $R_{L L}$ is much larger than $R_{L R}$, which means that the reflection almost maintains the incident polarization state for LHCP illumination, The opposite effect is found for $\phi=-25^{\circ}$. In both cases, the degree of circular polarization, $\mathrm{DOCP}=$ $2[(\mathrm{RHCP}-\mathrm{LHCP}) /(\mathrm{RHCP}+\mathrm{LHCP})]$, is $\sim \pm 1.8$.

We also study the metasurface DOCP under elliptical illumination conditions by varying $\phi$ and by controlling the

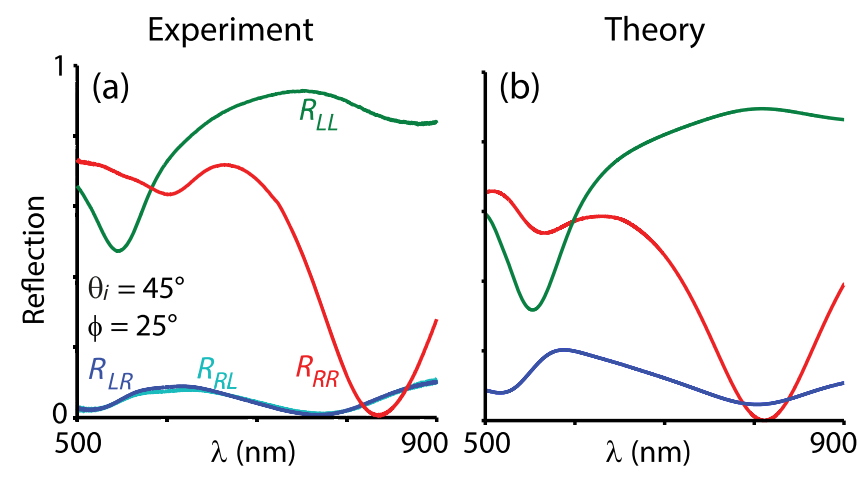

FIG. 6. Polarization properties of the reflected light. (a) Experimental and (b) calculated circular polarization components of the reflected light at $\theta_{i}=45^{\circ}$ and $\phi=25^{\circ}$. 
phase lag between the incident $p$ and $s$ components by careful rotation of an achromatic quarter-wave plate (Thorlabs AQWP05M-630). Calculations, using the same parameters as in Fig. 3, show that there are two narrow ranges of elliptically polarized light that trigger a rapid change of the DOCP at $\lambda_{r}$. These phase singularities occur at $\phi= \pm 25^{\circ}$ for a phase lag of $65^{\circ}$ and $245^{\circ}$, as indicated by circles in Fig. 7(a). We experimentally verify the position and general characteristics of the singularities [Figs. 6(b)-6(e)]. Indeed, the DOCP maintains large absolute values at $\phi= \pm 25^{\circ}$ and $\lambda_{r}$, and it is quite insensitive

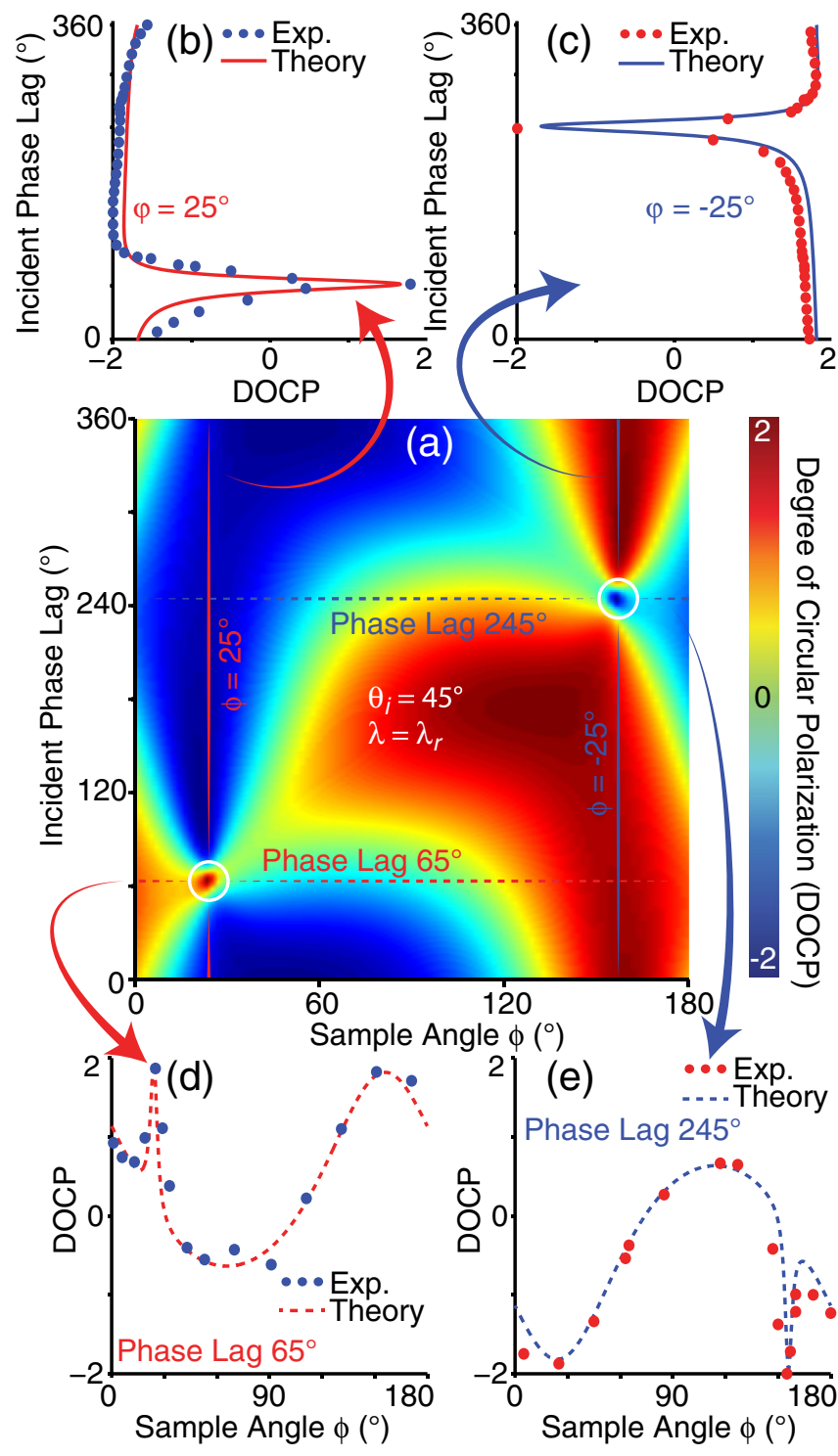

FIG. 7. Polarization properties of the reflected light under elliptical illumination. (a) Theoretical surface plot of the degree of circular polarization (DOCP) at $\lambda_{r}$ as function of $\phi$ and phase lag between the incident $p$ and $s$ polarizations. The DOCP at $\lambda_{r}$ as function of (b),(c) the phase lag and (d),(e) $\phi$. The horizontal and vertical lines in (a) correspond to the data in (b)-(e). The markers denote experimental data, and the lines are calculations. to the polarization state of the illumination except within a narrow range where the DOCP varies rapidly [Figs. 6(b) and 6(c)]. Similarly, we observe a discontinuity in the DOCP as a function of $\phi$ [Figs. 6(d) and 6(e)] when the metasurface is illuminated with certain elliptical polarizations. This effect is due to the phase differences between the reflection coefficients together with the ellipticity of the illumination, resulting in destructive interference and a very low overall reflection (the net reflection coefficient at the singularity is $\sim 1 \%$ ). We also investigate the spectral properties at various $\phi$ for varying elliptically polarized illumination states. The results show that the singularity is, at least, three dimensional, that is, singular with respect to $\phi, \lambda$, and the incident polarization state, in accordance with our analytical model. These data are displayed and further discussed in Supplemental Material Sec. S7 [25].

\section{CONCLUSIONS}

In summary, we experimentally demonstrate that excitation of an anisotropic metasurface at an incidence angle above $\theta_{c}$ can lead to near-complete photon spin selectivity in the reflected light. This result is based on the hypothesis that the high asymmetry of the projected in-plane electrical fields generated for $\theta_{i}>\theta_{c}$, combined with the metasurface anisotropy, should lead to enhanced chiroptical effects. We obtain direct experimental evidence supporting the hypothesis by measuring reflections above and below $\theta_{c}$. $\Delta R$ is found to be completely reversible with sample rotation from $\phi$ to $-\phi$; that is, the two "enantiomeric" forms of the metasurface are accessible by a simple sample rotation or change of illumination direction. However, despite the large spin selectivity, the average optical chirality enhancement in the near fields of the nanoparticles is found to be small. The polarization state of the reflected light maintains a large degree of circular polarization under most polarization states of the illumination, and shows an interesting singularitylike switching behavior for certain illumination conditions.

The type of metasurface discussed here can be easily tuned and further optimized by, for instance, varying the nanoparticle geometry [25]. To generate a large $\Delta R$, the two in-plane resonances need to be spectrally well separated, which for gold results in large chiroptical effects down to $650 \mathrm{~nm}$, corresponding to an in-plane aspect ratio of 1.5. Based on calculations shown in Supplemental Material Sec. S4, this range can be extended to the blue by, for example, using silver as the plasmonic material [25]. Furthermore, the chiroptical bandwidth may be increased through a mixture of nanoparticle geometries on the surface, or by employing more lossy materials, although this may result in decreased overall performance.

Polarization manipulation has many intriguing uses in photonics and telecommunications. By controlling the polarization of light, these metasurfaces can be used for modulation purposes, routing, and holography [20,21,37]. 
The rapid DOCP switch makes this configuration a suitable refractometric sensor transducer, similarly to other phasesensitive refractive index plasmonic sensors $[31,38]$. The evanescent excitation could thereby pave the way for efficient chiroptical devices and new fascinating studies in a number of different fields of research.

\section{ACKNOWLEDGMENTS}

The authors acknowledge Professor P. Johansson and Dr. Z-J. Yang for complementary simulations and discussions, Dr. R. Verre for help with AFM measurements, and Dr. B. Sepúlveda for discussions. This work was supported by the Knut and Alice Wallenberg Foundation, the Swedish Foundation for Strategic Research (SSF), and the Chalmers Nanoscience and Nanotechnology Area of Advance.

[1] V. K. Valev, J. J. Baumberg, C. Sibilia, and T. Verbiest, Chirality and Chiroptical Effects in Plasmonic Nanostructures: Fundamentals, Recent Progress, and Outlook, Adv. Mater. 25, 2517 (2013).

[2] Y. Tang and A.E. Cohen, Optical Chirality and Its Interaction with Matter, Phys. Rev. Lett. 104, 163901 (2010).

[3] C. Leyder, M. Romanelli, J. Ph. Karr, E. Giacobino, T. C. H. Liew, M. M. Glazov, A. V. Kavokin, G. Malpuech, and A. Bramati, Observation of the Optical Spin Hall Effect, Nat. Phys. 3, 628 (2007).

[4] K. Konishi, M. Nomura, N. Kumagai, S. Iwamoto, Y. Arakawa, and M. Kuwata-Gonokami, Circularly Polarized Light Emission from Semiconductor Planar Chiral Nanostructures, Phys. Rev. Lett. 106, 057402 (2011).

[5] E. Hendry, T. Carpy, J. Johnston, M. Popland, R. V. Mikhaylovskiy, A. J. Lapthorn, S. M. Kelly, L. D. Barron, N. Gadegaard, and M. Kadodwala, Ultrasensitive Detection and Characterization of Biomolecules Using Superchiral Fields, Nat. Nanotechnol. 5, 783 (2010).

[6] M. Hentschel, M. Schaeferling, T. Weiss, N. Liu, and H. Giessen, Three-Dimensional Chiral Plasmonic Oligomers, Nano Lett. 12, 2542 (2012).

[7] J. K. Gansel, M. Thiel, M.l S. Rill, M. Decker, K. Bade, V. Saile, G. von Freymann, S. Linden, and M. Wegener, Gold Helix Photonic Metamaterial as Broadband Circular Polarizer, Science 325, 1513 (2009).

[8] R. Ogier, Y. Fang, M. Svedendahl, P. Johansson, and M. Käll, Macroscopic Layers of Chiral Plasmonic Nanoparticle Oligomers from Colloidal Lithography, ACS Photonics 1, 1074 (2014).

[9] X. Yin, M. Schäferling, B. Metzger, and H. Giessen, Interpreting Chiral Nanophotonic Spectra: The Plasmonic Born Kuhn Model, Nano Lett. 13, 6238 (2013).

[10] Y. Zhao, M. A. Belkin, and A. Alu, Twisted Optical Metamaterials for Planarized Ultrathin Broadband Circular Polarizers, Nat. Commun. 3, 870 (2012).

[11] S. C. Jiang, X. Xiong, Y. S. Hu, Y. H. Hu, G. B. Ma, R. W. Peng, C. Sun, and M. Wang, Controlling the Polarization
State of Light with a Dispersion-Free Metastructure, Phys. Rev. X 4, 021026 (2014).

[12] S.-C. Jiang, X. Xiong, Y.-S. Hu, S.-W. Jiang, Y.-H. Hu, D.-H. Xu, R.-W. Peng, and M. Wang, High-Efficiency Generation of Circularly Polarized Light via SymmetryInduced Anomalous Reflection, Phys. Rev. B 91, 125421 (2015).

[13] N. Yu, F. Aieta, P. Genevet, M. A. Kats, Z. Gaburro, and F. Capasso, A Broadband, Background-Free Quarter-Wave Plate Based on Plasmonic Metasurfaces, Nano Lett. 12, 6328 (2012).

[14] T. Verbiest, M. Kauranen, Y. Van Rompaey, and A. Persoons, Optical Activity of Anisotropic Achiral Surfaces, Phys. Rev. Lett. 77, 1456 (1996).

[15] E. Plum, X. X. Liu, V. A. Fedotov, Y. Chen, D. P. Tsai, and N. I. Zheludev, Metamaterials: Optical Activity without Chirality, Phys. Rev. Lett. 102, 113902 (2009).

[16] T. Cao, C. Wei, L. Mao, and Y. Li, Extrinsic 2D Chirality: Giant Circular Conversion Dichroism from a MetalDielectric-Metal Square Array, Sci. Rep. 4, 7442 (2014).

[17] B. M. Maoz, A. Ben Moshe, D. Vestler, O. Bar-Elli, and G. Markovich, Chiroptical Effects in Planar Achiral Plasmonic Oriented Nanohole Arrays, Nano Lett. 12, 2357 (2012).

[18] A. Yokoyama, M. Yoshida, A. Ishii, and Y. K. Kato, Giant Circular Dichroism in Individual Carbon Nanotubes Induced by Extrinsic Chirality, Phys. Rev. X 4, 011005 (2014).

[19] K. Y. Bliokh, A. Y. Bekshaev, and F. Nori, Extraordinary Momentum and Spin in Evanescent Waves, Nat. Commun. 5, 3300 (2014).

[20] R. Mitsch, C. Sayrin, B. Albrecht, P. Schneeweiss, and A. Rauschenbeutel, Quantum State-Controlled Directional Spontaneous Emission of Photons into a Nanophotonic Waveguide, Nat. Commun. 5, 5713 (2014).

[21] J. Petersen, J. Volz, and A. Rauschenbeutel, Chiral Nanophotonic Waveguide Interface Based on Spin-Orbit Interaction of Light, Science 346, 67 (2014).

[22] O. Hosten and P. Kwiat, Observation of the Spin Hall Effect of Light via Weak Measurements, Science 319, 787 (2008).

[23] F. J. Rodriguez-Fortuno, G. Marino, P. Ginzburg, D. O'Connor, A. Martinez, G. A. Wurtz, and A. V. Zayats, Near-Field Interference for the Unidirectional Excitation of Electromagnetic Guided Modes, Science 340, 328 (2013).

[24] D. O'Connor, P. Ginzburg, F. J. Rodriguez-Fortuno, G. A. Wurtz, and A. V. Zayats, Spin-Orbit Coupling in Surface Plasmon Scattering by Nanostructures, Nat. Commun. 5, 5327 (2014).

[25] See Supplemental Material at http://link.aps.org/ supplemental/10.1103/PhysRevX.5.041019 for $\theta$ dependence of $\Delta R$, near-field plots, and more details on the analytical theory.

[26] H. Fredriksson, Y. Alaverdyan, A. Dmitriev, C. Langhammer, D. S. Sutherland, M. Zaech, and B. Kasemo, Hole-Mask Colloidal Lithography, Adv. Mater. 19, 4297 (2007).

[27] D. Bedeaux and J. Vlieger, Optical Properties of Surfaces (Imperial College Press, London, 2001).

[28] A. Mendoza-Galvan, K. Jarrendahl, A. Dmitriev, T. Pakizeh, M. Käll, and H. Arwin, Optical Response of Supported Gold Nanodisks, Opt. Express 19, 12093 (2011). 
[29] M. Svedendahl and M. Käll, Fano Interference between Localized Plasmons and Interface Reflections, ACS Nano 6 , 7533 (2012).

[30] M. Svedendahl, P. Johansson, and M. Käll, Complete Light Annihilation in an Ultrathin Layer of Gold Nanoparticles, Nano Lett. 13, 3053 (2013).

[31] M. Svedendahl, R. Verre, and M. Käll, Refractometric Biosensing Based on Optical Phase Flips in Sparse and Short-Range-Ordered Nanoplasmonic Layers, Light Sci. Appl. 3, e220 (2014).

[32] D. M. Lipkin, Existence of a New Conservation Law in Electromagnetic Theory, J. Math. Phys. (N.Y.) 5, 696 (1964).

[33] M. Schäferling, X. Yin, and H. Giessen, Formation of Chiral Fields in a Symmetric Environment, Opt. Express 20, 26326 (2012).

[34] K. Hiramatsu, M. Okuno, H. Kano, P. Leproux, V. Couderc, and Hiro-o Hamaguchi, Observation of Raman Optical Activity by Heterodyne-Detected Polarization-Resolved
Coherent Anti-Stokes Raman Scattering, Phys. Rev. Lett. 109, 083901 (2012).

[35] A. García-Etxarri and J. A. Dionne, Surface-Enhanced Circular Dichroism Spectroscopy Mediated by Nonchiral Nanoantennas, Phys. Rev. B 87, 235409 (2013).

[36] M. Finazzi, P. Biagioni, M. Celebrano, and L. Duò, Quasistatic Limit for Plasmon-Enhanced Optical Chirality, Phys. Rev. B 91, 195427 (2015).

[37] L. Huang, X. Chen, H. Muehlenbernd, H. Zhang, S. Chen, B. Bai, Q. Tan, G. Jin, K.-W. Cheah, C.-W. Qiu, J. Li, T. Zentgraf, and S. Zhang, Three-Dimensional Optical Holography Using a Plasmonic Metasurface, Nat. Commun. 4, 2808 (2013).

[38] V. G. Kravets, F. Schedin, R. Jalil, L. Britnell, R. V. Gorbachev, D. Ansell, B. Thackray, K. S. Novoselov, A. K. Geim, A. V. Kabashin, and A. N. Grigorenko, Singular Phase Nano-optics in Plasmonic Metamaterials for Label-Free Single-Molecule Detection, Nat. Mater. 12, 304 (2013). 